\title{
Advanced Concept Ramjet Propulsion System Utilizing In-Situ Positron Antimatter Derived from Ultra-Intense Laser with Fundamental Performance Analysis
}

\author{
Robert Le Moyne1, Timothy Mastroianni² \\ ${ }^{1}$ Independent Author (Senior Member AIAA \& Senior Member IEEE), Running Springs, California, USA \\ ${ }^{2}$ Independent Author, Pittsburgh, Pennsylvania, USA \\ Email: rlemoyne07@gmail.com
}

Received December 2013

\section{Abstract}

The fundamental performance analysis of an advanced concept ramjet propulsion system using antimatter is presented. Antimatter is generated by ultra-intense laser pulses incident on a gold target. The scientific foundation for the generation of antimatter by an ultra-intense laser was established in the early 1970's and later demonstrated at Lawrence Livermore National Laboratory from 2008 to 2009 . Antimatter on the scale of $2 \times 10^{10}$ positrons were generated through a $\sim 1 \mathrm{ps}$ pulse from the Lawrence Livermore National Laboratory Titan laser that has an intensity of $\sim \mathbf{1 0}^{20}$ $\mathrm{W} / \mathrm{cm}^{2}$. The predominant mechanism is the Bethe-Heitler process, which involves high-energy bremsstrahlung photons as a result of electron-nuclei interaction. Propulsion involving lasers through chemical rather than non-chemical interaction has been previously advocated by Phipps. The major utilities of the ultra-intense laser derived antimatter ramjet are the capability to generate antimatter without a complex storage system and the ability to decouple the antimatter ramjet propulsion system from the energy source. For instance the ultra-intense laser and energy source could be terrestrial, while the ramjet could be mounted to a UAV as a propulsion system. With the extrapolation of current technologies, a sufficient number of pulses by ultra-intense lasers are eventually anticipated for the generation of antimatter to heat the propulsive flow of a ramjet. Fundamental performance analysis is provided based on an ideal ramjet derivation that is modified to address the proposed antimatter ramjet architecture.

\section{Keywords}

Ultra-Intense Laser, Antimatter, Positron, Antimatter Propulsion, Antimatter Generation, Ramjet Propulsion

\section{Introduction}

Over the past four decades both applied and theoretical science have progressively evolved to yield the capacity

How to cite this paper: Le Moyne, R. and Mastroianni, T. (2014) Advanced Concept Ramjet Propulsion System Utilizing In-Situ Positron Antimatter Derived from Ultra-Intense Laser with Fundamental Performance Analysis. Journal of Applied Mathematics and Physics, 2, 19-26. http://dx.doi.org/10.4236/jamp.2014.25003 
to generate on demand and in-situ antimatter from ultra-intense lasers incident on high atomic number targets [1-3]. The fruition of ultra-intense derived antimatter generation enables a novel conceptual strategy for ramjet propulsion. The theory and application of antimatter generated from an ultra-intense laser is presented. Other propulsion systems using antimatter and non-chemical ramjet propulsion architectures are contrasted to the proposed antimatter ramjet. The utility of the ramjet system and history is briefly presented, and the analytical basis for an ideal ramjet is derived. The ideal ramjet analysis is amended to accommodate the ramjet propulsion system utilizing in-situ antimatter. The profile of thrust for the antimatter-derived ramjet is presented as a function of Mach number. Matters of technology feasibility are contrasted to other historic technology extrapolations. The objective of the following publication is to present a ramjet propulsion system utilizing in-situ antimatter from the perspective of fundamental performance analysis. The antimatter ramjet propulsion system could be eventually integrated into a UAV as a propulsion application.

\section{A Quantum Leap in Antimatter Generation, the Implementation of an Ultra-Intense Laser}

During the early 1970's Shearer et al. presented a perspective for the generation of positrons derived from the incidence of lasers on the intensity order of approximately $10^{20} \mathrm{~W} / \mathrm{cm}^{2}$ [1]. Experiments conducted throughout 2008 to 2009 confirmed the capacity to generate considerable quantities of antimatter positrons through the application of an ultra-intense laser on a high atomic number target, such as gold [2,3]. A noticeable utility of the ultra-intense laser generation of antimatter is the capacity to produce antimatter on demand, in contrast to traditional antimatter propulsion architectures that require the considerable complexity of a correlated storage system.

Lawrence Livermore National Laboratory during the span of 2008 to 2009 experimentally demonstrated the ability to generate antimatter through an ultra-intense short pulse laser incident on a gold target. The laser intensity was on the order of $10^{20} \mathrm{~W} / \mathrm{cm}^{2}$, based on pulses of approximately $1 \mathrm{ps}$. The ultra-intense laser pulse generated $2 \times 10^{10}$ positrons, and about $90 \%$ of the positrons were discharged aft to the laser target. The laser target consisted of about $1 \mathrm{~mm}$ thick of gold, which is considered a high atomic number target $[2,3]$. The basis for the generation of antimatter as a consequence of the incident ultra-intense laser pulse is derived from the resultant interaction of electrons and nuclei [2-4]. In particular two processes are considered the foundation for the generation of antimatter through a high atomic number nuclei: Trident process and Bethe-Heitler process [2,4].

The Trident process represents a single step for the generation of the antimatter positron. In essence the electron directly interacts with the nuclei to yield an electron-positron pair.

$$
e^{-}+Z \rightarrow e^{+}+2 e^{-}+Z
$$

$e^{+}:$Positron

$e^{-}:$Electron

$Z$ : High atomic number nuclei(such as gold) $[2,4]$.

The Bethe-Heitler process involves two steps. First, high-energy bremsstrahlung photons are produced by fast electrons. Second, bremsstrahlung photons interact with the nuclei yielding electron-positron pairs.

$$
\begin{gathered}
e^{-}+Z \rightarrow \gamma+e^{-}+Z \\
\gamma+Z \rightarrow e^{+}+e^{-}+Z
\end{gathered}
$$

$\gamma:$ Bremsstrahlung photons [2,4].

Although the Trident process only consists of one step, the Bethe-Heitler process predominates with a cross section of 100 times greater than the Trident process. The target thickness also influences the predominant electron-positron pair generation process. The Bethe-Heitler process is dominant for gold targets on the order of $1 \mathrm{~mm}$ thick; by contrast the Trident process is the prevalent for gold targets that are about 3.5 micrometers thick [4].

The Lawrence Livermore National Laboratory experiment generated $2 \times 10^{10}$ positrons based on $\sim 1$ ps pulse was accomplished using the Titan laser. The Titan laser has an intensity of $\sim 10^{20} \mathrm{~W} / \mathrm{cm}^{2}$, wavelength of 1054 $\mathrm{nm}$, and energy of $\sim 120 \mathrm{~J}$. The Bethe-Heitler process was identified as the predominant mechanism with positron temperature of about $2 \mathrm{MeV}$. Approximately 10 times as many positrons were anisotropically ejected normal to the aft relative to the frontof the target. The gold target was $1 \mathrm{~mm}$ thick $[2,3]$. 
Other concepts incorporating the use of a laser as the basis for a propulsion system have been researched, developed, tested, and evaluated. Phipps has developed a method for laser ablation propulsion, which has historically evolved over the course of three decades. Laser ablation propulsion incorporates either a continuous wave laser or pulsed laser, which produces thrust based on generated plasma or vapor from a condensed matter target [5].

The implications of the proposed antimatter ramjet enable a decoupling of the propulsion system and the respective energy source. Traditionally the energy source of a propulsion system requires fuel stored in a fuel tank system, which can expand the propulsion system complexity [6,7]. However the antimatter ramjet configuration conveys an ultra-intense laser to a gold target on the ramjet. The ultra-intense laser source could in principle be the size of a building, and the power source could be on the scale of a conventional power plant. Yet the gold target with the incident ultra-intense laser could be geometrically consistent with a ramjet propulsion system on the scale of a UAV.

The proposed antimatter ramjet propulsion system should be contrasted to existing magnetic confinement antimatter concepts and other non-chemical ramjet propulsion systems. Space propulsion applications innovated by Kammash involving antimatter also associate with complex magnetic confinement and storage of the antimatter [8]. However, the proposed antimatter ramjet propulsion system generates and subsequently annihilates antimatter based on controlled pulsing of the ultra-intense laser. Complex magnetic confinement is unnecessary for the antimatter ramjet configuration.

The other non-chemical ramjet propulsion system is an interstellar ramjet conceptualized by Bussard during 1960. The Bussard interstellar ramjet utilizes interstellar gas as a propulsive energy source. The interstellar ramjet involves nuclear fusion at relativistic speeds [9]. Note that the Bussard interstellar ramjet was conceptualized over a half century ago, but not yet developed for application. The interstellar ramjet significantly differs from the antimatter ramjet propulsion system. The antimatter ramjet propulsion system is intended for a terrestrial application.

Other air breathing propulsion systems, such as a turbojet, could be incorporated in the above antimatter generation strategy. However, the ramjet characteristically consists of minimal moving parts [7]. The ramjet configuration is selected for the conceptual antimatter propulsion concept, due to the minimal complexity. The origins of the ramjet propulsion system date from the dawn of the $20^{\text {th }}$ century and have progressively evolved over the course of the past century [10].

\section{Historical Foundation of the Ramjet and Derivation of the Ideal Ramjet}

The concept of the ramjet was initiated by Lake through a patent during 1909 [10]. Four decades later the ramjet was flight tested by Renee Leduc in 1949 through the Leduc 010. A transport aircraft was required to achieve sufficient flight velocity and altitude for the Leduc 010 ramjet system [11]. The ramjet propulsion system is not capable of generating static thrust. Therefore, a booster system is required [10]. The analytical framework of the ideal ramjet with propulsive force provided by antimatter annihilation is presented. Ideal ramjet analyses for a variety of ramjet configurations, including rocket-ramjet combined cycles, have been successfully applied by LeMoyne [12-15].

The ramjet constitutes minimal complexity, regarding air-breathing propulsion, since the ramjet consists of minimal moving parts. Compression of the airflow occurs at the inlet, instead of through rotating machinery. There are three major components of the ramjet: the diffuser, combustion chamber, and nozzle. For ideal ramjet performance analysis, the ramjet is delineated into six stations. The diffuser section represents stations 1 to 2 , and the airflow undergoes compression. At the combustion chamber, which is represented by stations 2 to 4 , the ramjet fuel is injected and mixed with the airflow, resulting in combustion. The expansion of the ramjet propulsive flow occurs in the nozzle section, which is represented by stations 4 to 6 . Based on the six-station representation for the diffuser, combustion chamber, and nozzle for the ramjet, the ideal ramjet performance analysis can be derived based on isentropic derivations [7].

The thrust of an ideal ramjet is derived with the assumption that combustion products undergo isentropic expansion through the nozzle and the nozzle expands the propulsive flow to an exit pressure equal to the ambient pressure.

$$
F=\dot{m}_{a}\left[(1+f) u_{e}-u_{a}\right]+\left(P_{e}-P_{a}\right) A_{e}
$$




$$
P_{e}=P_{a}
$$

$P_{e}:$ Exit pressure

$P_{a}:$ Ambient pressure

$A_{e}:$ Exit area

$F:$ Thrust

$$
F=\dot{m}_{a}\left[(1+f) u_{e-} u_{a}\right]
$$

$u_{e}$ : Exit velocity

$u_{a}$ : Ambient airflow velocity

$$
f=\frac{\dot{m}_{f}}{\dot{m}_{a}}: \text { Fuel }- \text { air ratio }
$$

$m_{f}:$ Fuel mass flow

$m_{a}$ : Ambient air mass flow [7].

The stagnation pressure is assumed constant throughout the ramjet stations. Compression and expansion of the flow is isentropic. Heat and mass addition at the combustion chamber occur at low velocity and constant pressure. The molecular weight averaged ideal gas constant and specific heat ratio are assumed constant through the ramjet stations. The flight Mach number and nozzle exit Mach number are considered equal, because of the equivalent stagnation pressure and static pressure ratio.

$$
\begin{gathered}
\frac{P_{O a}}{P_{a}}=\left(1+\frac{k-1}{2} M_{a}^{2}\right)^{k /(k-1)} \\
\frac{P_{O e}}{P_{e}}=\left(1+\frac{k-1}{2} M_{e}^{2}\right)^{k /(k-1)} \\
\frac{P_{O a}}{P_{a}}=\frac{P_{O e}}{P_{e}} \\
M_{a}=M_{e}
\end{gathered}
$$

$P_{O e}:$ Exit stagnation pressure

$P_{O a}:$ Ambient airflow stagnation pressure

$M_{a}:$ Flight Mach number

$M_{e}$ : Exit Mach number

$k:$ Specific heat ratio [7].

The exhaust velocity can be derived, since the flight Mach number and exit Mach number have been determined equivalent.

$$
\begin{aligned}
& u_{e}=\frac{a_{e}}{a_{a}} u_{a} \\
& a_{e}=\sqrt{k R T_{e}} \\
& a_{a}=\sqrt{k R T_{a}}
\end{aligned}
$$

$a_{e}:$ Exit speed of sound

$a_{a}:$ Airflow speed of sound

$$
u_{e}=\sqrt{\frac{T_{e}}{T_{a}}} u_{a}
$$

$T_{e}:$ Static exit temperature

$T_{a}$ : Static airflow temperature(ambient [7].

Because the flight Mach number and exit Mach number are equivalent, the ratios of the stagnation and static 
temperatures are equivalent. The expansion from station 4 of the combustion chamber is isentropic to the nozzle exit section. Therefore, exit stagnation temperature and station 4 combustion chamber stagnation temperature are equivalent.

$$
\left.u_{e}=\sqrt{\frac{T_{O 4}}{T_{O a}}} u_{a}\right)
$$

$T_{\mathrm{O} 4}$ : Stagnation exit temperature $T_{\mathrm{Oa}}$ : Stagnation airflow temperature(ambient) [7].

Based on these assumptions for the ideal ramjet, thrust as a function of ambient air mass flow can be derived.

$$
\frac{F}{m_{a}}=M_{a} \sqrt{k R T_{a}}\left[(1+f) \sqrt{\frac{T_{O 4}}{T_{a}}}\left(1+\frac{k-1}{2} M_{a}^{2}\right)^{-1 / 2}-1\right]
$$

[7].

\section{Derivation of an Ideal Ramjet-Using Ultra-Intense Laser Derived In-Situ Antimatter}

The primary difference between the conventional ideal ramjet and the ideal ramjet using in-situ ultra-intense laser derived antimatter is the fuel air ratio will be set to zero. The stagnation temperature of the combustion chamber is obtained using an energy balance. The energy balance involves the incoming flow stagnation temperature at station 2 with antimatter annihilation per kilogram of airflow determining the stagnation temperature at station 4 .

$$
Q_{\text {antimatter }}=c_{p}\left(T_{O 4}-T_{O 2}\right)
$$

$Q_{\text {antimatter }}:$ Antimatter annihilation heat

addition per kilogram of airflow

$c_{p}:$ Constant pressure specific heat

$T_{O 2}$ : Stagnation temperature station 2 .

The stagnation temperature of station 4 should be derived as a function of Mach number.

$$
\frac{T_{O}}{T}=1+\frac{k-1}{2} M^{2}
$$

$T_{O}:$ Stagnation temperature

$T$ : Static temperature [6].

The energy balance relations yield the flowing equation.

$$
T_{04}=\frac{Q_{\text {antimatter }}}{c_{p}}+T_{a}\left(1+\frac{k-1}{2} M^{2}\right)
$$

The combustion chamber heat addition is based on in-situ antimatter annihilation derived from an ultra-intense laser system that is incident on a $1 \mathrm{~mm}$ thick gold target. The following ideal ramjet thrust equation is derived for an antimatter ideal ramjet using ultra-intense laser derived in-situ antimatter with the fuel air ratio set to zero.

$$
\frac{F}{m_{a}}=M_{a} \sqrt{k R T_{a}}\left[\sqrt{\frac{\frac{Q_{\text {antimatter }}}{c_{p}}+T_{a}\left(1+\frac{k-1}{2} M^{2}\right)}{T_{a}}}\left(1+\frac{k-1}{2} M_{a}^{2}\right)^{-1 / 2}-1\right]
$$

\section{In-Situ Antimatter Derived from an Ultra-Intense Laser}

The Titan laser of Lawrence Livermore National Laboratory is an ultra-intense laser $\left(10^{20} \mathrm{~W} / \mathrm{cm}^{2}\right)$ with $\sim 1 \mathrm{ps}$ pulses that has demonstrated the capacity to generate $2 \times 10^{10}$ positrons incident on a $\sim 1 \mathrm{~mm}$ thick gold target. Approximately $90 \%$ of the positrons are emitted anisotropic and aft to the laser target through predominantly the 
Bethe-Heitler process, which is resultant of interaction between electrons and the nuclei through bremsstrahlung photons. Therefore, each $\sim 1$ ps pulse yields $1.8 \times 10^{10}$ positrons to the aft of the laser target with about $2 \mathrm{MeV}$ of kinetic energy [2,3].

The rest mass of the positron and electron pair is $2 \mathrm{mc}^{2}=1.02 \mathrm{MeV}$, and $\mathrm{m}$ is the electron rest mass equivalent to the positron rest mass and $\mathrm{c}$ is the speed of light. The positron kinetic energy is $2 \mathrm{MeV}$. Assuming the collisions are elastic, such that kinetic energy is conserved, the energy of a positron and electron annihilation is $3.02 \mathrm{MeV}$ [16,17]. For each ultra-intense laser $\sim 1$ ps pulse $8.71 \times 10^{-6} \mathrm{~kJ}$ of energy is released aft to the laser target. Extrapolating the current state of technology, up to $1.8 \times 10^{22}$ positrons could be emitted aft to the laser target. The laser intensity threshold for the direct process exceeds the Titan laser researched at Lawrence Livermore National Laboratory by eight orders of magnitude. Future extrapolations of technology are anticipated to increase laser energy and pulse rate [2].

\section{Model and Results for Ramjet Propulsion Involving Ultra-Intense Laser Derived In-Situ Antimatter}

The antimatter ramjet model assumed future extrapolations and improvements in laser energy, pulse rate, and the possibility for multiple ultra-intense lasers to generate antimatter. A total of $10^{8}$ pulses on the picosecond time scale using an ultra-intense laser $\left(10^{20} \mathrm{~W} / \mathrm{cm}^{2}\right)$ are assumed. The antimatter annihilation produces $871 \mathrm{~kJ}$ aft to the laser target and adjacent to the ramjet propulsive flow. For the flight profile, the ambient air mass flow is scaled to $1 \mathrm{~kg} / \mathrm{s}$. A profile addressing the antimatter ramjet thrust as a function of Mach number is presented. The ambient conditions of Table $\mathbf{1}$ are incorporated into the model.

As illustrated in Figure 1, the optimal thrust occurs in the supersonic regime. The antimatter ramjet produces a maximum thrust of $471 \mathrm{~N}$ at a Mach number of 2.77; and the antimatter annihilation achieves a station 4 stagnation temperature of $1494 \mathrm{~K}$. Based on the model results, the antimatter ramjet propulsion application would be suitable for integration with a UAV propulsion system. The thrust is directly proportional to the number of ultra-intense laser pulses. Given the scalable nature of the ultra-intense laser derived antimatter generation, the antimatter ramjet propulsion system is also applicable for Micro-UAV propulsion applications $[6,18]$.

\section{Engineering Challenges and Technology Extrapolations for Ultra-Intense Laser Derived Antimatter Ramjet Propulsion}

Regarding the future feasibility of ultra-intense laser derived antimatter propulsion; some historic comparatives should be addressed, such as the extrapolation of aircraft technologies. The scientific foundation for flight can be attributed to Bernoulli's insight, dated to roughly the mid-1700s. With the progressive evolution of technologies, such as the internal combustion engine, powered flight became a reality a century and a half later in the early 1900's. Roughly four decades later, during the late 1940's, supersonic flight was achieved with further extrapolations of propulsion technologies [6]. The ramjet was conceptualized in the first decade of the 1900's and later flight-tested four decades later in the later 1940's [10,11]. Goddard developed rocketry in the mid-1920s, and by the later 1960's travel to the moon by rocketry was a reality $[6,19]$.

By logical comparison of technology extrapolation, consider the implementation of ultra-intense lasers for generating antimatter. Shearer et al. conceptualized the original scientific foundation for ultra-intense laser generation of antimatter during the early 1970's [1]. However, roughly three and a half decades later, scientists at Lawrence Livermore National Laboratory successfully generated antimatter through an ultra-intense laser [2,3].

Table 1. Ambient flight conditions for antimatter ramjet.

\begin{tabular}{cc}
\hline Flight altitude $(\mathrm{m})$ & 6000 \\
Ambient temperature $(\mathrm{K})$ & 249.20 \\
Ambient pressure $\left(\mathrm{N} / \mathrm{m}^{2}\right)$ & 47217 \\
Constant pressure specific heat $(\mathrm{kJ} / \mathrm{kg}-\mathrm{K})$ & 1.01 \\
Specific heat ratio & 1.4 \\
Molecular weight averaged gas constant $(\mathrm{J} /(\mathrm{kg}-\mathrm{K}))$ & 287 \\
\hline
\end{tabular}




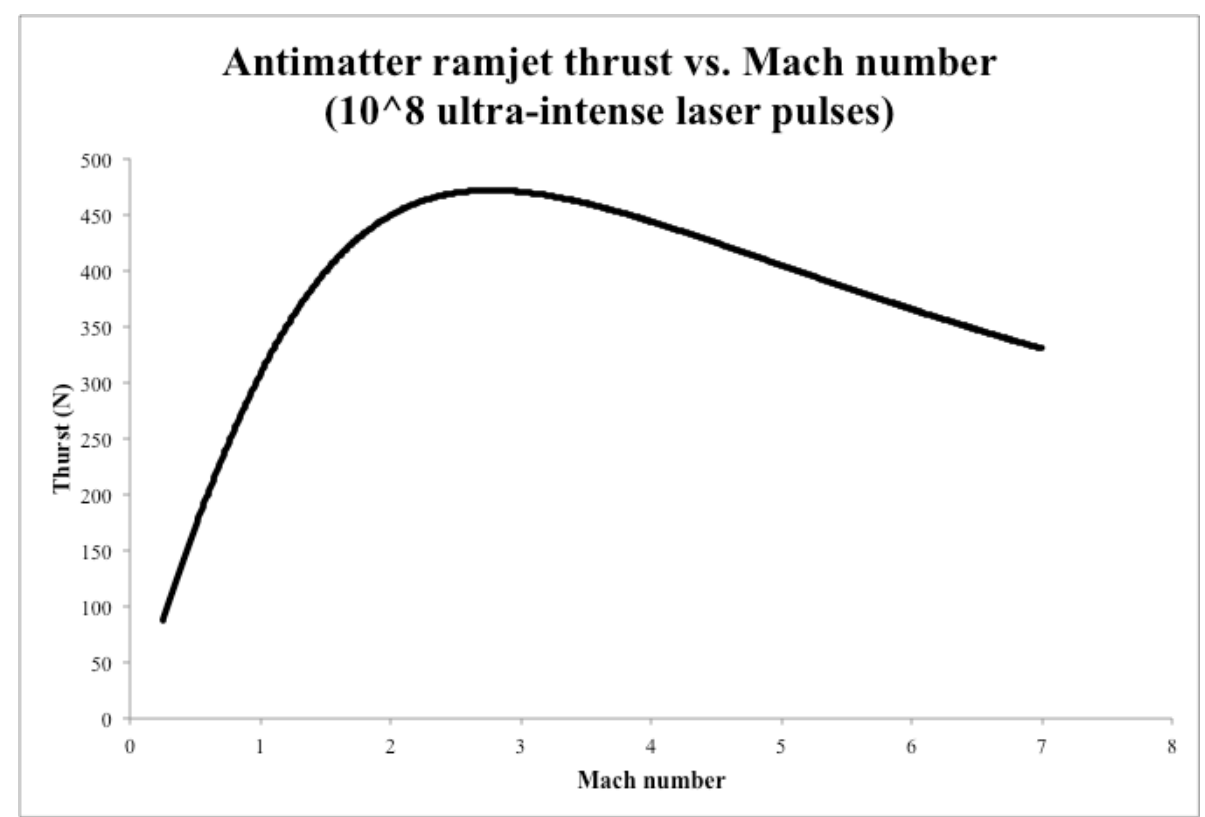

Figure 1. Antimatter ramjet thrust as a function of flight Mach number.

Future extrapolations of ultra-intense laser applications involve increases in laser energy and pulse rate. The threshold for the direct process regarding laser intensity is eight orders of magnitude greater than the Lawrence Livermore National Laboratory Titan laser [2]. Currently, tabletop scale lasers have been demonstrated to impart energy to electrons on the $\mathrm{MeV}$ scale [20].

Of course, for the progressive extrapolation of the concept of ultra-intense laser derived antimatter ramjet propulsion to reach fruition, multiple issues must be addressed. The optimal conditions for the antimatter annihilation should be considered. The optimal density for absorbing resultant antimatter annihilation radiation should be determined. The optimal flight altitude and influence of humidity should be addressed, especially with respect to the absorptivity and emissivity characteristics of the ultra-intense laser. Given the potentially relativistic energies imparted by the ultra-intense laser, relativity relations, such as time dilation and length contraction should be included while configuring the geometry of the antimatter ramjet propulsion system. The ramjet was selected because of the simplicity of the configuration, but other air breathing configurations should be also contrasted.

In the future, the geometric constraints of the current Titan ultra-intense laser are anticipated to be progressively miniaturized. Laser energy levels and pulse rate for ultra-intense lasers are projected to increase. The incorporation of multiple ultra-intense lasers may also address the number of laser pulses to achieve antimatter generation sufficient to provide propulsive energy for a ramjet. The major utility of the ultra-intense laser derived antimatter propulsion system is the decoupling of the energy source for propulsion and the ramjet itself. The ultra-intense laser derived antimatter propulsion configuration can operate with a terrestrial and stationary energy source to power the ultra-intense laser, which can also be stationary and terrestrial.

\section{Conclusion}

The thrust as a function of Mach number was presented for an ultra-intense laser derived in-situ antimatter ramjet through fundamental performance analysis using an ideal ramjet. Maximum thrust of $471 \mathrm{~N}$ and corresponding Mach number of 2.77were identified. Over the course of three and a half decades, the generation of antimatter through ultra-intense lasers has evolved from theory by Shearer et al. during the early 1970's to application during 2008 to 2009 by the Titan laser of Lawrence Livermore National Laboratory.

The ultra-intense laser derived antimatter concept implicates the capability to decouple the actual propulsion system from the actual energy source of the propulsive flow. A terrestrial positioned power source and ultra-intense laser system can convey the laser energy to an in-flight ramjet application without the need for an antimatter storage system. Antimatter is generated as a function of the incident ultra-intense laser on a gold target. The 
antimatter ramjet configuration could be applied to a UAV propulsion system.

Design challenges, such as absorption of resultant antimatter annihilation radiation, optimization of flight characteristics, and analysis analytical consideration of the potentially relativistic effects of the resultant antimatter should be addressed. For example, the restricting bounds of an ultra-intense laser incident to a gold target generating positrons are unknown. The future improvements in the evolution of ultra-intense lasers are anticipated, such as improvements in laser energy, pulse rate, and further convergence to the direct process threshold of laser intensity. An example of the progressive evolution of chemically inherent laser propulsion is demonstrated by Phipps, which spanned the course of three decades from theory to application. Another example of the aerospace/aeronautical evolutionary potential can be elucidated through the evolution of flight from powered flight to supersonic flight over the course of less than a half-century.

\section{References}

[1] Shearer, J.W., Garrison, J., Wong, J. and Swain, J.E. (1973) Pair Production by Relativistic Electrons from an Intense Laser Focus. Physical Review A, 8, 1582-1588. http://dx.doi.org/10.1103/PhysRevA.8.1582

[2] Chen, H., Wilks, S.C., Bonlie, J.D., Chen, S.N., Cone, K.V., Elberson, L.N., Gregori, G., Meyerhofer, D.D., Myatt, J., Price, D.F., Schneider, M.B., Shepherd, R., Stafford, D.C., Tommasini, R., Van Maren, R. and Beiersdorfer, P. (2009) Making Relativistic Positrons Using Ultraintense Short Pulse Lasers. Physics of Plasmas, 16.

[3] Chen, H., Wilks, S.C., Bonlie, J.D., Liang, E.P., Myatt, J., Price, D.F., Meyerhofer, D.D. and Beiersdorfer, P. (2009) Relativistic Positron Creation Using Ultraintense Short Pulse Lasers. Physical Review Letters, 102. http://dx.doi.org/10.1103/PhysRevLett.102.105001

[4] Nakashima, K. and Takabe, H. (2002) Numerical Study of Pair Creation by Ultraintense Lasers. Physics of Plasmas, 9, 1505-1512. http://dx.doi.org/10.1063/1.1464145

[5] Phipps, C., Birkan, M., Bohn, W., Eckel, H. A., Horisawa, H., Lippert, T., Michaelis, M., Rezunkov, Y., Sasoh, A., Schall, W., Scharring, S. and Sinko, J. (2010) Review: Laser-Ablation Propulsion. Journal of Propulsion and Power, 26, 609-637. http://dx.doi.org/10.2514/1.43733

[6] Anderson, J.D. (1989) Introduction to Flight. McGraw-Hill, New York.

[7] Hill, P.G. and Peterson, C.R. (1992) Mechanics and Thermodynamics of Propulsion. Addison-Wesley, New York.

[8] Kammash, T. and Galbraith, D. (1990) Antimatter Induced Fusion Rocket Propulsion. Proceedings of AIAA, ASME, SAE, and ASEE 26th Joint Propulsion Conference, AIAA 1990-2509, Orlando, Florida.

[9] Bussard, R.W. (1960) Galactic matter and interstellar flight. Astronautica Acta, 6, 179-194.

[10] Waltrup, P.J., White, M.E., Zarlingo, F. and Gravlin, E.S. (2002) History of U.S. Navy Ramjet, Scramjet, and MixedCycle Propulsion Development. Journal of Propulsion and Power, 18, 14-27. http://dx.doi.org/10.2514/2.5928

[11] http://aerostories.free.fr/constructeurs/leduc/page8.html

[12] LeMoyne, R. (2008) Fundamental Analysis of a Combined Cycle Rocket-Ramjet Propulsion System. Proceedings of 15th AIAA International Space Planes and Hypersonic Systems and Technologies Conference, AIAA 2008-2617, Dayton, Ohio.

[13] LeMoyne, R. (2009) Fundamental Analysis of a Tandem LH2-LOX Rocket-Ramjet Propulsion System. Proceedings of the AIAA Space 2009 Conference and Exposition, AIAA-2009-6658, Pasadena, California.

[14] LeMoyne, R. (2009) Fundamental Analysis of a Combined Cycle Hydrocarbon-Liquid Oxygen Tandem RamjetRocket Propulsion System. Proceedings of 16th AIAA/DLR/DGLR International Space Planes and Hypersonic Systems and Technologies Conference, AIAA-2009-7335, Bremen.

[15] LeMoyne, R. (2011) Fundamental Analysis of a First Stage Ramjet-Rocket Combined Cycle and Second Stage Hydrolysis Propulsion System with Trajectory Weighting. Proceedings of 17th AIAA International Space Planes and Hypersonic Systems and Technologies Conference AIAA-2011-2333, San Francisco. http://dx.doi.org/10.2514/6.2011-2333

[16] Serway, R.A., Moses, C.J. and Moyer, C.A. (1989) Modern Physics. Saunders College, Philadelphia, Pennsylvania.

[17] Serway, R.A. (1990) Physics for Scientists and Engineers. Saunders College, Philadelphia, Pennsylvania.

[18] Turns, S.R. (1996) An Introduction to Combustion: Concepts and Applications. McGraw-Hill, New York.

[19] Sutton, G.P. (2006) History of Liquid Propellant Rocket Engines. AIAA, Reston, Virginia.

[20] Gahn, C., Tsakiris, G.D., Pretzler, G., Witte, K.J., Thirolf, P., Habs, D., Delfin, C. and Wahlström, C.G. (2002) Generation of MeV Electrons and Positrons with Femtosecond Pulses from a Table-Top Laser System. Physics of Plasmas, 9, 987-999. http://dx.doi.org/10.1063/1.1446879 